https://doi.org/10.48009/2_iis_2006_213-217

\title{
WHY VISITORS LEAVE WEB SITES WITHOUT BUYING: TOWARD A UNIFIED THEORY OF WEB SITE DESIGN
}

\author{
Voraphan Manomuth, Utah State University, voraphanm@cc.usu.edu \\ John F. Vinsonhaler, Utah State University, john.vinsonhaler@usu.edu \\ Gerry W. Scheffelmaier, Middle Tennessee State University, gwscheff@ mtsu.edu
}

\begin{abstract}
First, the current theory, minimal cognitive processing theory $(M C P T)$ is summarized. The MCPT applies Newell and Simon's cognitive processing theory [1] from human computer interaction (HCI) to user behavior on retail Web sites. It proposes that the two latent variables are responsible for a user staying on or leaving a Web site. Clickstream cost is the amount of cognitive processing required to reach a given page in the Web site, which is operationally defined by counting the number of Web site features and applying the Hick Hyman law. Personal cost limit is the total amount of cognitive processing that the visitor is willing to invest in the Web site. When the clickstream cost exceeds the personal cost limit, the visitor exits unless he/she has already found and purchased the desired product. From this it is deduced that the major latent variable responsible for Web site abandonment is Web site complexity (amount of cognitive processing required by the Web site). Second, an empirical study evaluating the MCPT is summarized. In this experimental study, two Web sites were created: a low-complexity Web site (with minimal features) and a high-complexity Web site (with all of the features provided as defaults by the Web site building software osCommerce). The Web sites were otherwise identical. In total, 120 participants (each buying a product on both Web sites) were used. Four major hypotheses were deduced from the MCPT: the higher the Web site complexity, (a) the lower the number of products found and purchased, $(b)$ the greater the amount of time required for purchase, (c) the greater the abandonment rate, and (d) the lower the user satisfaction. All major hypotheses were confirmed with $p=.000$. Secondary analyses suggest an effect of knowledge of B2C Web site design, self-reported experience in Web purchasing, and a learning effect across Web sites, as suggested by the MCPT. Third, plans for generalizing are summarized.
\end{abstract}

Keywords: E-Commerce Web Sites, Web Site Evaluation, Cognition, Human Computer Interaction

\section{INTRODUCTION}

Currently, Web site design that is thought to improve buyer conversion is driven by lists of features based on expert opinion, anecdotal accounts of Web analytics studies, and proprietary Web analytics studies that perform statistical analyses of user behavior on Web sites. We could find no report of a theory that links empirically effective Web design features to generalized theoretic principles that are related to research and theories in other disciplines. The inclusion of theory would considerably add to the coherence of the Web site design discipline.

\section{MINIMAL COGNITIVE PROCESSING THEORY}

The MCPT [12] posits that unnecessary complexity of Web site leads to an unnecessary cognitive processing (CP) load, which causes user dissatisfaction and pre-purchase exit from the Web site. The MCPT is strongly influenced by the works of Krug [7] and Nielsen [9]. In particular, the Hick Hyman law from the human computer interaction model $[4,5]$ has heavily influenced the MCPT. The Hick Hyman law suggests that complexity leads to increased cognitive processing. As a Web site becomes more complex, more cognitive processing is required in the purchasing process and the more likely it is that the user will give up and exit the Web site without purchasing. The MCPT assumes that Internet shoppers are pressed for time and are impatient with the $\mathrm{CP}$ required in traversing a highcomplexity Web site. In other words, a user has a limit on how much CP he/she will tolerate when he/she attempts to make online purchases. When this user's personal cost limit is exceeded, he/she exit the Web site without buying.

\section{Assumptions of the MCPT}

There are three assumptions of the MCPT as follows. First, the cognitive processing model applies to web buyers. Second, clickstream cost and a user's personal cost limit determine when a user exits the Web site without purchasing. Third, measuring Web 
page or Web site complexity and cognitive processing is as a valid measurement.

In this discussion, it is assumed that visitors have a goal of buying products. Thus, the type and number of products can be manipulated as an independent variable. If several products are used, it would be assumed they are different products, otherwise learning would certainly affect the amount of $\mathrm{CP}$.

\section{Complexity of Web Pages and Web sites}

Cognitive processing of Web page is defined as the amount of $\mathrm{CP}$ demanded by the Web page. Complexity of the Web page is the set of observable Web page features which ultimately determines the amount of $\mathrm{CP}$ required by the Web page for any given group of users. Once it is operationally defined, it may be used to manipulate the amount of $\mathrm{CP}$ as an independent variable.

A Web page feature is a Web site design element which is perceivable to the visitor and which (a) provides access to functionality, for example by taking the user to another Web page or (b) provides a structure for the Web page, permitting the organization of other features.

Complexity of the Web site is the sum of the complexities of its Web pages that is the number of clickable and non-clickable features. It is believed that applying the Hick Hyman law to Web page complexity gives a reasonable estimate of the amount of $\mathrm{CP}$ that is required by the Web page and is given by the following:

Amount of Cognitive Processing $=\log _{2}(N)$, (1)

where $N=$ the number of stimuli (features) on the Web page and where all the stimuli (features) are equally probable. In this case, they all have a probability equaling one since they appear every time the page is loaded. It is also assumed that the total amount of cognitive processing of a Web site is the sum of the amount of cognitive processing of the component Web pages. Similar applications to choice behavior on Web pages have been made by [8] and others. The time it takes to make a decision is roughly proportional to the amount of $\mathrm{CP}$ that the user has expended to make a decision and the entropy of the decision (the $\log$ of the number of alternatives).

\section{RESEARCH QUESTIONS}

Our analysis of the MCPT raises several research questions which the study, described below, was designed to address:

(1) Will a higher amount of Web site complexity cause a lower number of products found and purchased? It might be argued that this is obvious: "sure, more complex Web sites are more difficult to use." One might apply the same argument to Newton [6]: "well sure, everything rolls downhill." Until now, the possible relationship between Web site complexity and purchase success is based on anecdotal evidence from Web Analytics researchers. Further, there has been no theoretical basis for this hypothesis. A controlled experiment was assembled where complexity was theoretically connected to cognitive processing (a research concept common to many fields) using established HCI research. Web site complexity was objectively measured by counting Web site features while purchasing success was objectively measured by counting the number of successful purchases in a given time period. Further, there is an implicit quantitative relationship between Web site complexity and purchasing, which could be utilized if resources were available to add treatment groups for other values of complexity. Besides clear empirical predictions, a good theory leads to generalization and suggests a path for research and theory development.

(2) Will a higher amount of Web site complexity cause greater amount of time required for purchase? There is no obvious reason why complex Web sites should require more time, unless an assumption is made about the relationship between Web site complexity and cognitive processing: the more complexity, the more cognitive processing required and the more time required to buy a product. In this case the cognitive processing is linearly related to the logarithm to the base two of the complexity (Hick Hyman Law). Because of the many HCI studies (even from Positron Emission Tomography (PET) studies a brain function for simple and complex tasks [11]), this is probably a sound assumption.

(3) Will a higher amount of Web site complexity cause lower user satisfaction? Why should Web site complexity decrease satisfaction? Satisfaction with the Web site is a global judgment based on at least three judgments: how easy it is to purchase from the Web site, the coherence of its content, and ease of navigation. It would appear to the authors that all of these judgments are impacted 
by the amount of cognitive processing demanded by the user.

(4) Will a higher amount of Web site complexity cause higher abandonment rate? When the clickstream cost exceeds the user's personal cost limit, he/she exits the Web site. Over a group of participants, a Web site demanding high cognitive processing will yield a higher cognitive processing cost for any clickstreams, which will exceed the personal cost limit for more users.

(5) Is there any evidence that users build longterm memory of Web sites and use these memory structures to reduce the cognitive processing load? The MCPT is based upon the theory of cognitive processing which assumes long-term memory cognitive schema are constructed in short-term memory during the processing of a Web site, and later brought back to short-term memory to aid with later page processing. If the user has experience with many B2C Web sites, one would expect that a general knowledge of the conventional Web site design would reduce cognitive processing required in Web sites with a similar design. The more experience with B2C Web sites, the higher should be the number of products found and purchased and the lower the amount of time required for purchase should be.

\section{EMPIRICAL STUDY}

In order to assess the MCPT, we gathered data from 120 students attending the College of Business at Utah State University. This study was a traditional experimental design that involved two Web sites. In this study, there was one independent variable with two values: the low-complexity Web site, which has the fewest number of characteristics linked to increase cognitive processing required on each Web page (e.g., amount of unnecessary text); and the highcomplexity Web site, which has the most numbers of characteristics linked to increase cognitive processing required on each Web page. The main dependent variables were (a) number of products found and purchased; (b) abandonment rate; (c) amount of time required for purchase (in the testing processes in the main study, a maximum time of 15 minutes was used for each Web site and a 5- or 15-minute maximum time limit was used for scoring number of products found and purchased); and (d) user satisfaction score. In university computer labs, small groups of 30 participants were tested for each of four conditions. Participants were randomly assigned to one of the four booklet conditions. Four conditions were assigned with a stratified random procedure to each booklet based on a random numbers table and were counterbalanced over Case 1 and Case 2. A case consisted of a Web site and a problem (e.g., find two specific products to purchase). Each data collection booklet consisted of two cases (two Web sites and two problems).

After the motivation instruction was given, each participant completed the following steps: (a) each participant filled out the demographic questionnaire and the knowledge test of conventional Web site design; (b) each participant was instructed to buy two specific products on a specific Web site; (c) each participant reported the entrance and exit times as posted on the top of the home page and on the credit card error page, which is the equivalence of a purchase (which were checked by the experimenter or assistant); (d) each participant completed the postexperimental session questionnaire (satisfaction questionnaire) after the case; (e) the participants then repeated steps two through four for the second case; and (f) a retest for the satisfaction questionnaire was administered at the end of the experiment.

\section{Satisfaction Questionnaires}

The scale items were developed using the theoretical foundation provided by [2] to measure the user satisfaction. In some cases, the items reflect an adaptation of existing scale items, while other items were developed specifically for this study using the theoretical domains outlined by prior research $[1,3$, 10]. The satisfaction questionnaire consisted of a $7-$ point Likert scale, with $1=$ "Strongly Disagree" and 7 = "Strongly Agree" as anchors. The questionnaire contained questions regarding the purchase experience that the participant had with the assigned Web site. To control for error attributable to the direction of items, ten items were positively worded, whereas two items were negatively worded. Scores from the questionnaire were used to measure the participant's evaluation of the high-complexity and the low-complexity Web sites. The purpose of the questionnaire was to assess which Web sites users preferred (as compared to measures indicating which Web site was more satisfying).

\section{Statistical Analyses}

The following five major statistical analyses were used in this study: a paired-samples $t$-test; an independent-samples $t$-test, a chi-square test, a bivariate correlation, and reliability analyses. All major hypotheses were confirmed with $p=.000$. Two of the secondary research hypotheses were also supported while the other two hypotheses were partially supported. 


\section{RESULTS}

Scale Reliability. The 12-item satisfaction scale obtained a Cronbach's alpha reliability of 0.972 . Further, test scores for the satisfaction questionnaire account for 94 percent $\left(r^{2}=(.969)^{2}=.938\right)$ of the variability in re-test scores for the questionnaire.

For the main research hypotheses, all of the theoretical predictions were supported. From the results (see Table 1), for RH1—as Web site complexity increases, number of products found and purchased decreases, all of the results were statistically significant with $p=.000$ in both the pairsamples $t$-test and the chi-square test analyses, therefore confirming the theoretical prediction of RH1. Second, for RH2 - as Web site complexity increases, the amount of time required for purchase increases, all of the results was statistically significant with $p=.000$ in the paired-samples $t$-test analysis; therefore, confirming the theoretical prediction of RH2. Third, for RH3-as Web site complexity increases, user satisfaction decreases, all of the results were statistically significant with $p=$
.000 in the paired-samples $t$-test, therefore confirming the theoretical prediction of RH3. Fourth, for RH4-as Web site complexity increases, abandonment rate increases, all of the results were statistically significant with $p=.014$ in both the pairsamples $t$-test and chi-square test analyses, therefore confirming the theoretical prediction of RH4. For the secondary research hypotheses, all the theoretical predictions were supported. From the findings, the result from the analysis was statistically significant in the chi-square test, Pearson's correlation analyses, therefore confirming the theoretical prediction that "the more experience with $\mathrm{B} 2 \mathrm{C}$ Web sites (the more knowledge of typical Web site design), the lower the amount of time required for purchase and the greater the number of products found and purchased." For the learning effect (number of products found and purchased \& amount of time required for purchase on the first Web site presented vs. number of products found and purchased $\&$ amount of time required for purchase on the second Web site presented), the results were statistically significant with $p=.000$ in the paired-samples $t$-test analyses, therefore confirming that a learning effect occurred.

Table 1. Statistical Analyses of the Findings

\begin{tabular}{ll}
\hline RH \# & Statistical Analyses \\
\hline RH1 & Paired-Samples $t$-test: \\
& $\begin{array}{l}\text { Using a 5- minute time limit, } t(119)=8.98, p=.000, d=1.2 \\
\text { Chi-Square Test: }\end{array}$ \\
& Using a 5- minute time limit, $x^{2}(1)=62.127, p=.000, P h i^{2}=0.26$ \\
RH2 & $\frac{\text { Paired-Samples } t \text { Test: }}{\text { Using 114 participants, } t(113)=10.25, p=.000, d=1.4}$ \\
RH3 & $\frac{\text { Paired-Samples } t \text { Test: }}{\text { The total score user satisfaction, } t(119)=13.52, p=.000, d=1.5}$ \\
RH4 & $\frac{\text { Paired-Samples } t \text { Test: }}{\text { Using a 15-minute time limit, } t(119)=2.503, p=.014, d=0.3}$ \\
& $\frac{\text { Chi-Square Test: }}{\text { Using a 15-minute time limit, } x^{2}(1)=6.15, p=.013, P h i^{2}=0.026}$ \\
\hline
\end{tabular}

\section{CONCLUSIONS}

Based on the findings from the main and secondary hypotheses, all of the predictions from the MCPT have been confirmed in this study. However, this is only one study and offers no guarantee that other studies will replicate the findings. Nevertheless, this is a very promising first study for this theory.

\section{Implications of the MCPT}

First, the more cognitive processing required by a Web site to purchase a product, the lower the number of products found and purchased and the higher the abandonment rate. Second, the longer the time required to find and purchase a product, the lower the number of products found and purchased. Third, adding more features to a Web site will not necessarily improve buyer conversion rate. Forth, for experienced web buyers, conventionally designed Web sites should yield a higher number of products found and purchased than non-conventional Web sites. Fifth, the more experience with B2C Web sites, the lower the amount of time required for purchase and the better the number of products found and purchased. 


\section{Limitations}

First, the participants were assigned tasks to better control the design. Second, the participants in the study simulated purchases; they did not actually buy real products with real money. Third, this was a college student population, but it clearly had more experience with Web purchasing than was anticipated in the main study. Fourth, the variety of products on the Web site was more limited than can be found on many commercial B2C Web sites, which may have affected the results. Fifth, many B2C Web sites have much more complex pages and menus. This might have increased all of effects of the independent variable. The absence of a search engine was due to fact that the theory has not been developed to predict the impact of this type of feature.

\section{Further Research}

One clear next step would be to replicate the study and use real purchasing instead of simulated purchasing. As a theory, the MCPT only handles half of the problem of why people stay on or leave a Web site. Staying on the Web site long enough to purchase is determined by two latent variables: clickstream cost and personal cost limit. To extend the theory, assumptions must be added about the Web site determiners of the personal cognitive processing limit (how long the user is willing to work on the Web site). Clearly, these determiners include price of products, quality of products, the amount of information needed to make a decision, product involvement, motivation, and so forth. Adding these assumptions should improve the predictions and make the theory more useful to practitioners.

\section{REFERENCES}

1. Chin, J. P., Diehl, V. A., \& Norman, K. L. (1988). Development of an instrument measuring user satisfaction of the human-computer interface. Paper presented at the SIGCHI conference on Human factors in computing systems, Washington, DC.

2. Davis, F. D., Bagozzi, R. P., \& Warshaw, P. R. (1989). User acceptance of computer technology:
A comparison of two theoretical models. Management Science, 35(8), 982.

3. Escalas, J. E., Jain, K., \& Strebel, J. E. (2001). Satisfaction, frustration, and delight: A framework for understanding how consumers interact with Web sites. In O. Lee (Ed.), Internet marketing research: Theory and Practice (pp. 231-251). Hershey, PA: Idea Group Publishing.

4. Hick, W. E. (1952). On the rate of gain of information. Quarterly Journal of Experimental Psychology, 4, 11-26.

5. Hyman, R. (1953). Stimulus information as a determinant of reaction time. Journal of Experimental Psychology, 45, 188-196.

6. JOC/EFR. (2000). Sir Isaac Newton. Received December 5, 2005, from http://wwwhistory.mcs.standrews.ac.uk/Mathematicians/Newton.html

7. Krug, S. (2005). Don't make me think: A common sense approach to Web usability (2nd ed.). Berkeley, CA: New Riders.

8. Landauer, T. K., \& Nachbar, D. W. (1985). Selection from alphabetic and numeric menu trees using a touch screen: Breath, depth, and width. Paper presented at the Proceedings of the SIGCHI conference on Human factors in Computing Systems., San Francisco, CA.

9. Nielsen, J. (2000). Designing Web usability: The practice of simplicity. Indianapolis, IN: New Riders.Proctor, R. W., \& Vu, K. L. (2003). Human information processing: An overview for HCI. In J. M. Carroll (Ed.), HCI models, theories, and frameworks: Toward a multidisciplinary science. San Francisco, CA: Morgan Kaufmann.

10. Scheffelmaier, G. W., Vinsonhaler, J., \& Paper, D. (2004). Purchase time and user satisfaction as effected by Web site convenience. Issue in Information Systems, 1, 294-300.

11. Valk, P. E., Bailey, D. L., Townsend, D. W., \& Maisey, M. N. (2003). Positron Emission Tomography: Basic science and clinical practice. London: Springer-Verlag.

12. Vinsonhaler, J. F. (2006). The minimal cognitive processing theory. Unpublished manuscript. (Available from the author, john.vinsonhaler@usu.edu). 